Elect. Comm. in Probab. 15 (2010), 299-313

\title{
SPECTRAL NORM OF CIRCULANT TYPE MATRICES WITH HEAVY TAILED ENTRIES
}

\author{
ARUP BOSE ${ }^{1}$ \\ Stat-Math Unit, Indian Statistical Institute, 203 B. T. Road Kolkata 700108, India \\ email: bosearu@gmail.com \\ RAJAT SUBHRA HAZRA \\ Stat-Math Unit, Indian Statistical Institute, 203 B. T. Road Kolkata 700108, India \\ email: rajat_r@isical.ac.in \\ KOUSHIK SAHA ${ }^{2}$ \\ Stat-Math Unit, Indian Statistical Institute, 203 B. T. Road Kolkata 700108,India \\ email: koushik_r@isical.ac.in
}

Submitted October 8, 2009, accepted in final form June 15, 2010

AMS 2000 Subject classification: Primary 60B20; Secondary 15B52, 60B10, 15A60, 60 F05

Keywords: Large dimensional random matrix, eigenvalues, Toeplitz matrix, circulant matrix, symmetric circulant matrix, reverse circulant matrix, spectral norm, moving average process, power transfer function.

\begin{abstract}
We first study the probabilistic properties of the spectral norm of scaled eigenvalues of large dimensional Toeplitz, circulant and symmetric circulant matrices when the input sequence is independent and identically distributed with appropriate heavy tails.

When the input sequence is a stationary two sided moving average process of infinite order, we scale the eigenvalues by the spectral density at appropriate ordinates and study the limit for their maximums.
\end{abstract}

\section{Introduction}

Matrices with suitable patterned random inputs where the dimension tends to infinity are known as large dimensional random matrices. In this article we focus on the (symmetric) Toeplitz, circulant, reverse circulant and symmetric circulant matrices defined as follows: let $\left\{Z_{0}, Z_{1}, \ldots\right\}$ be a sequence of real random variables, which will be called the input sequence.

(Symmetric) Toeplitz matrix $T_{n}$. This $n \times n$ symmetric matrix with input $\left\{Z_{i}\right\}$ is the matrix with $(i, j)$-th entry $Z_{|i-j|}$ for all $i, j$. Toeplitz matrices appear as the covariance matrix of stationary processes, in shift-invariant linear filtering and in many aspects of combinatorics, time series and har-

\footnotetext{
${ }^{1}$ RESEARCH SUPPORTED BY J.C.BOSE NATIONAL FELLOWSHIP, DEPARTMENT OF SCIENCE AND TECHNOLOGY, GOVERNMENT OF INDIA

${ }^{2}$ RESEARCH SUPPORTED BY CSIR FELLOWSHIP, GOVT. OF INDIA.
} 
monic analysis. [Bai(1999)] proposed the study of the large Toeplitz matrix with independent inputs. For results in the non random situation we refer the reader to [Grenander and Szegö(2001)]. Circulant matrix $C_{n}$. The first row is $\left(Z_{0} Z_{1} \ldots Z_{n-1}\right)$ and the $(j+1)$-th row is obtained by giving its $j$-th row a right circular shift by one position. This is not a symmetric matrix and its $(i, j)$-th element is given by $Z_{(j-i+n) \bmod n}$.

One of the usefulness of the circulant matrix is due to its deep connection to the Toeplitz matrix. The former has an explicit easy formula for its eigenvalues. The spectral analysis of the latter is much harder and challenging. If the input $\left\{Z_{l}\right\}_{l \geq 0}$ is square summable, then the circulant matrix approximates the corresponding Toeplitz matrix in several senses when the dimension grows. See [Gray (2006)] for a recent and relatively easy account.

The circulant matrices are diagonalized by the Fourier matrix $F=\left(\left(F_{s, t}\right)\right), F_{s, t}=e^{2 \pi i s t / n}, 0 \leq s, t<$ $n$. Their eigenvalues are the discrete Fourier transform of the input sequence $\left\{a_{l}\right\}_{0 \leq l<n}$ and are given by $\lambda_{t}=\sum_{l=0}^{n-1} a_{l} e^{-2 \pi i t / n}, 0 \leq t<n$. The eigenvalues of the circulant matrices arise crucially in time series analysis. For instance, the periodogram of a sequence $\left\{a_{l}\right\}_{l \geq 0}$ is defined as $n^{-1}\left|\sum_{l=0}^{n-1} a_{l} e^{2 \pi i j / n}\right|^{2},-\left\lfloor\frac{n-1}{2}\right\rfloor \leq j \leq\left\lfloor\frac{n-1}{2}\right\rfloor$ and is a straightforward function of the eigenvalues of the corresponding circulant matrix. The study of the properties of the periodogram is fundamental in the spectral analysis of time series. See for instance [Fan and Yao(2003)]. The maximum of the perdiogram, in particular, has been studied in [Davis and Mikosch(1999)]. Some recent developments on random circulant matrices are available in [Meckes (2009)] and [Bose et al.(2009a)].

Symmetric circulant matrix $S C_{n}$. This matrix is a symmetric version of the usual circulant matrix. The first row $\left(Z_{0} Z_{1} Z_{2} \ldots Z_{2} Z_{1}\right)$ is a palindrome and the $(j+1)$-th row is obtained by giving its $j$-th row a right circular shift by one position. Its $(i, j)$-th element is given by $Z_{n / 2-|n / 2-| i-j||}$.

When the input sequence is i.i.d. with positive variance, then it is no longer square summable. In that case, the spectral behaviour of the symmetric circulant and the symmetric Toeplitz are quite different. Compare for example, the limiting spectral distribution results of [Bose and Mitra(2002)] and [Massey et al.(2007)] for the symmetric circulant matrix, and of [B Bryc, Dembo and Jiang (2006)] and [Hammond and Miller(2005)] for the Toeplitz matrix.

On the other hand, consider the random symmetric band Toeplitz matrix, where the banding parameter $m$, which essentially is a measure of the number of nonzero entries, satisfies $m \rightarrow \infty$ and $m / n \rightarrow 0$. Then again its spectral distribution is approximated well by the corresponding banded symmetric circulant matrix. See for example [Kargin(2009)] and [Basak and Bose(2009)].

Reverse circulant matrix $R C_{n}$. The first row of this matrix is $\left(Z_{0} Z_{1} \ldots Z_{n-1}\right)$ and the $(j+1)$-th row is obtained by giving its $j$-th row a left circular shift by one position. This is a symmetric matrix and its $(i, j)$-th entry is given by $Z_{(i+j-2) \bmod n}$. This matrix arises in various applications of time series. The eigenvalue structure of this matrix is very closely related to the periodogram of the input sequence. The LSD of the reverse circulant was derived in [Bose and Mitra(2002)]. This has been used in the study of the symmetric band Hankel matrices. See [Basak and Bose(2009)] for details.

Spectral norm. The spectral norm $\|A\|$ of a matrix A with complex entries is the square root of the largest eigenvalue of the positive semidefinite matrix $A^{*} A$ :

$$
\|A\|=\sqrt{\lambda_{\max }\left(A^{*} A\right)}
$$

where $A^{*}$ denotes the conjugate transpose of $A$. Therefore if $A$ is an $n \times n$ real symmetric matrix or $A$ is a normal matrix then

$$
\|A\|=\max _{1 \leq i \leq n}\left|\lambda_{i}\right|
$$

where $\lambda_{1}, \lambda_{2}, \ldots, \lambda_{n}$ are the eigenvalues of $A$. 
Existing limit results for the spectral norm. For the existing limit results on spectral norms of the Toeplitz and circulant matrices, see [Bose and Sen(2007)], [Meckes(2007)], [Bryc and Sethuraman(2009)], [Bose et al.(2009b)] and [Adamczak(2010)]. For spectral norm and radii of non-central random matrices see [Silverstein(1994)]. The maximum eigenvalues of the Wigner and the sample covariance matrix have been extensively studied, see [Bai and Yin (1988)] and [Yin et al.(1988)] for details. All these works are for the situation when the entries have finite moment of at least order two.

[Soshnikov (2004)] shows the distributional convergence of the maximum eigenvalue of an appropriately scaled Wigner matrix with heavy tailed entries $\left\{x_{i j}\right\}$ satisfying $\mathrm{P}\left(\left|x_{i j}\right|>x\right)=h(x) x^{-\alpha}$ where $h$ is a slowly varying function at infinity (that is, $h(t x) / h(x) \rightarrow 1$ as $x \rightarrow \infty$ ) and $0<\alpha<2$. The limiting distribution is $\Phi_{\alpha}(x)=\exp \left(-x^{-\alpha}\right)$. A similar result was proved for the sample covariance matrices in [Soshnikov (2006)] with Cauchy entries. These results on the Wigner and the sample covariance matrices were extended in [Auffinger et al.(2009)] to the case $0 \leq \alpha \leq 4$.

Our results. We focus on the above listed four matrices when the input sequence is heavy tailed, and $0<\alpha<1$. We establish the distributional convergence of the spectral norm of the three circulant matrices. Though we are unable to obtain the exact limit in the Toeplitz case, we provide upper and lower bounds. Our approach is to exploit the structure of the matrices and use existing methods on the study of maximum of periodograms for heavy tailed sequences.

It seems to be a nontrivial problem to derive properties of the spectral norm in the case of moving average process inputs. We resort to scaling each eigenvalue by the power transfer function (defined in Section 3) at the appropriate ordinate as described below and then consider their maximum. For any of the above mentioned matrix $A_{n}$, we define $\mathrm{M}\left(A_{n}, f\right)=\max _{1 \leq k \leq n} \frac{\left|\lambda_{k}\right|}{\sqrt{2 \pi f\left(\omega_{k}\right)}}$ where $f$ is the power transfer function corresponding to $\left\{x_{n}\right\}$ and $\left\{\lambda_{k}\right\}$ are the eigenvalues of $A_{n}$. Similar scaling has been used in the study of periodograms (see [Davis and Mikosch(1999)], [Mikosch et al.(2000)], [Lin and Liu(2009)]). We show the distributional convergence of $\mathrm{M}\left(A_{n}, f\right)$ for the three circulant matrices. Any general result without the scaling seems difficult to derive without further assumptions. However in this setup the results are immediate from the results on the spectral norm of their i.i.d. counterparts.

\section{Results for i.i.d. input}

Notation and preliminaries. Let $\left\{Z_{t}, t \in \mathbb{Z}\right\}$ be a sequence of i.i.d random variables with common distribution $F$ where $F$ is in the domain of attraction of an $\alpha$-stable random variable with $0<\alpha<1$. Thus, there exist $p, q \geq 0$ with $p+q=1$ and a slowly varying function $L(x)$, such that

$$
\lim _{x \rightarrow \infty} \frac{\mathrm{P}\left(Z_{1}>x\right)}{\mathrm{P}\left(\left|Z_{1}\right|>x\right)}=p, \lim _{x \rightarrow \infty} \frac{\mathrm{P}\left(Z_{1} \leq-x\right)}{\mathrm{P}\left(\left|Z_{1}\right|>x\right)}=q \text { and } \mathrm{P}\left(\left|Z_{1}\right|>x\right) \sim x^{-\alpha} L(x) \text { as } x \rightarrow \infty .
$$

A random variable $Y_{\alpha}$ is said to have a stable distribution $S_{\alpha}(\sigma, \beta, \mu)$ if there are parameters $0<\alpha \leq 2, \sigma \geq 0,-1 \leq \beta \leq 1$ and $\mu$ real such that its characteristic function has the form

$$
\mathrm{E}\left[\exp \left(i t Y_{\alpha}\right)\right]= \begin{cases}\exp \left\{i \mu t-\sigma^{\alpha}|t|^{\alpha}(1-i \beta \operatorname{sgn}(t) \tan (\pi \alpha / 2))\right\}, & \text { if } \quad \alpha \neq 1 \\ \exp \{i \mu t-\sigma|t|(1+(2 i \beta / \pi) \operatorname{sgn}(t) \ln |t|)\}, & \text { if } \quad \alpha=1\end{cases}
$$

If $\beta=\mu=0$, then $Y_{\alpha}$ is symmetric $\alpha$-stable $S \alpha S$. For details on stable processes see [Samorodnitsky and Taqqu (1994)]. In the description of our results, we shall need the following: let $\left\{\Gamma_{j}\right\},\left\{U_{j}\right\}$ and $\left\{B_{j}\right\}$ be three independent sequences defined on the same probability space where $\left\{\Gamma_{j}\right\}$ is the arrival sequence 
of a unit rate poisson process on $\mathbb{R}, U_{j}$ are i.i.d $U(0,1)$ and $B_{j}$ are i.i.d. satisfying

$$
\mathrm{P}\left(B_{1}=1\right)=p \text { and } \mathrm{P}\left(B_{1}=-1\right)=q,
$$

where $p$ and $q$ are as defined in (1). We also define

$$
Y_{\alpha}=\sum_{j=1}^{\infty} \Gamma_{j}^{-1 / \alpha} \sim S_{\alpha}\left(C_{\alpha}^{-\frac{1}{\alpha}}, 1,0\right) \text { where } C_{\alpha}=\left(\int_{0}^{\infty} x^{-\alpha} \sin x d x\right)^{-1} .
$$

For a nondecreasing function $f$ on $\mathbb{R}$, let $f^{\leftarrow}(y)=\inf \{s: f(s)>y\}$. Then the scaling sequence $\left\{b_{n}\right\}$ is defined as

$$
b_{n}=\left(\frac{1}{\mathrm{P}\left[\left|Z_{1}\right|>\cdot\right]}\right)^{\leftarrow}(n) \sim n^{1 / \alpha} L_{0}(n) \text { for some slowly varying function } L_{0} .
$$

Define

$$
\omega_{k}=\frac{2 \pi k}{n} \text { for } 0 \leq k \leq n \text {. }
$$

The reverse circulant and the circulant. The eigenvalues $\left\{\lambda_{k}, 0 \leq k \leq n-1\right\}$ of $b_{n}^{-1} R C_{n}$ are given by (see [Bose and Mitra(2002)]):

$$
\begin{cases}\lambda_{0} & =b_{n}^{-1} \sum_{t=0}^{n-1} Z_{t} \\ \lambda_{n / 2} & =b_{n}^{-1} \sum_{t=0}^{n-1}(-1)^{t} Z_{t}, \text { if } n \text { is even } \\ \lambda_{k}=-\lambda_{n-k} & =\sqrt{I_{n}\left(\omega_{k}\right)}, 1 \leq k \leq\left[\frac{n-1}{2}\right],\end{cases}
$$

where

$$
I_{n}\left(\omega_{k}\right)=\frac{1}{b_{n}^{2}}\left|\sum_{t=0}^{n-1} Z_{t} e^{-i t \omega_{k}}\right|^{2} .
$$

The eigenvalues of $b_{n}^{-1} C_{n}$ are given by

$$
\lambda_{j}=b_{n}^{-1} \sum_{t=1}^{n} Z_{t} e^{i t \omega_{j}}, \quad 0 \leq j \leq n-1 .
$$

Note that $\left\{\left|\lambda_{k}\right|^{2} ; 1 \leq k<n / 2\right\}$ is the periodogram of $\left\{Z_{i}\right\}$ at the frequencies $\left\{\omega_{k} ; 1 \leq k<n / 2\right\}$. From the eigenvalue structure of $C_{n}$ and $R C_{n}$, it is clear that $\left\|b_{n}^{-1} C_{n}\right\|=\left\|b_{n}^{-1} R C_{n}\right\|$ and therefore they have identical limiting behavior which is stated in the following result.

Theorem 1. Assume that the input sequence is i.i.d. $\left\{Z_{t}\right\}$ satisfying (1). Then for $\alpha \in(0,1)$, $\left\|b_{n}^{-1} C_{n}\right\| \Rightarrow Y_{\alpha}$ and $\left\|b_{n}^{-1} R C_{n}\right\| \Rightarrow Y_{\alpha}$, where $Y_{\alpha}$ is as in (3).

The symmetric circulant. The eigenvalues $\left\{\lambda_{k}, 0 \leq k \leq n-1\right\}$ of $b_{n}^{-1} S C_{n}$ are given by:

(i) for $n$ odd:

$$
\left\{\begin{array}{l}
\lambda_{0}=b_{n}^{-1}\left[Z_{0}+2 \sum_{j=1}^{[n / 2]} Z_{j}\right] \\
\lambda_{k}=b_{n}^{-1}\left[Z_{0}+2 \sum_{j=1}^{[n / 2]} Z_{j} \cos \left(\omega_{k} j\right)\right], \quad 1 \leq k \leq[n / 2]
\end{array}\right.
$$

(ii) for $n$ even:

$$
\left\{\begin{array}{l}
\lambda_{0}=b_{n}^{-1}\left[Z_{0}+2 \sum_{j=1}^{\frac{n}{2}-1} Z_{j}+Z_{n / 2}\right] \\
\lambda_{k}=b_{n}^{-1}\left[Z_{0}+2 \sum_{j=1}^{\frac{n}{2}-1} Z_{j} \cos \left(\omega_{k} j\right)+(-1)^{k} Z_{n / 2}\right], \quad 1 \leq k \leq \frac{n}{2}
\end{array}\right.
$$

with $\lambda_{n-k}=\lambda_{k}$ in both cases. 
Theorem 2. Assume that the input sequence is i.i.d. $\left\{Z_{t}\right\}$ satisfying (1). Then for $\alpha \in(0,1)$, $\left\|b_{n}^{-1} S C_{n}\right\| \Rightarrow 2^{1-1 / \alpha} Y_{\alpha}$, where $Y_{\alpha}$ is as in (3).

Remark 1. (i) Theorem 1 and 2 are rather easy to derive when $p=1$, that is, when the left tail is negligible compared to the right tail. Let us consider $\left\|b_{n}^{-1} R C_{n}\right\|$ and note from the eigenvalue structure that,

$$
\left\|b_{n}^{-1} R C_{n}\right\| \leq b_{n}^{-1} \sum_{t=1}^{n}\left|Z_{t}\right|
$$

For the lower bound note that

$$
P\left(\left\|b_{n}^{-1} R C_{n}\right\|>x\right) \geq P\left(\lambda_{0}>x\right)=P\left(b_{n}^{-1} \sum_{t=1}^{n} Z_{t}>x\right) .
$$

Now since $P\left(\left|Z_{1}\right|>x\right) \sim P\left(Z_{1}>x\right)$ as $x \rightarrow \infty$, the upper and lower bound converge with the same scaling constant and hence Theorem 1 holds. The details on these convergence can be found in Chapter 1 of [Samorodnitsky and Taqqu (1994)]. Similar conclusion can be drawn for the symmetric circulant matrices too when $p=1$.

(ii) When the input sequence $\left\{Z_{i}\right\}$ are i.i.d. non negative and satisfies (1) with $\alpha \in(1,2)$ then from above it is easy to derive the distributional behavior spectral norm. In particular if $k_{j}=$ $\frac{\alpha}{\alpha-1}\left(j^{\frac{\alpha-1}{\alpha}}-(j-1)^{\frac{\alpha-1}{\alpha}}\right)$ and $\widetilde{Y_{\alpha}}=\sum_{j=1}^{\infty}\left(\Gamma_{j}-k_{j}\right) \sim S_{\alpha}\left(C_{\alpha}^{-\frac{1}{\alpha}}, 1,0\right)$ then,

$$
P\left(\frac{\left\|R C_{n}\right\|-n \mathrm{E}\left[Z_{1}\right]}{b_{n}}>x\right) \rightarrow P\left(\widetilde{Y_{\alpha}}>x\right) \text { as } n \rightarrow \infty,
$$

and

$$
P\left(\frac{\left\|S C_{n}\right\|-n \mathrm{E}\left[Z_{1}\right]}{b_{n}}>x\right) \rightarrow P\left(2^{1-1 / \alpha} \widetilde{Y_{\alpha}}>x\right) \text { as } n \rightarrow \infty .
$$

When $\alpha=1$, and $\left\{Z_{i}\right\}$ are non negative

$$
\mathrm{P}\left(\frac{\left\|R C_{n}\right\|-n b_{n} \int_{0}^{\infty} \sin \left(\frac{x}{b_{n}}\right) P\left(Z_{1} \in d x\right)}{b_{n}}>x\right) \rightarrow P\left(\widetilde{\widetilde{Y}_{\alpha}}>x\right),
$$

where $\widetilde{\widetilde{Y}_{\alpha}}$ is a $S_{1}(2 / \pi, 1,0)$ random variable. Similar results hold for symmetric circulant matrices.

The Toeplitz. Resolving the question of the exact limit of the Toeplitz spectral norm seems to very difficult. Here we provide a good upper and lower bound in the distribution sense.

Theorem 3. Suppose that the input sequence is i.i.d. $\left\{Z_{t}\right\}$ satisfying (1). Then for $\alpha \in(0,1)$ and $\gamma>0$

$P\left(2 \sum_{j=1}^{\infty}\left(1-U_{j}\right) \Gamma_{j}^{-1 / \alpha}>\gamma\right) \leq \liminf _{n} P\left(b_{n}^{-1}\left\|T_{n}\right\|>\gamma\right) \leq \underset{n}{\limsup } P\left(b_{n}^{-1}\left\|T_{n}\right\|>\gamma\right) \leq P\left(2 \sum_{j=1}^{\infty} \Gamma_{j}^{-1 / \alpha}>\gamma\right)$.

Remark 2. The case when $\alpha \in[1,2)$ and $p \neq 1$ and $\left\{Z_{i}\right\}$ are not necessarily non negative appears to be a non trivial problem. In the reverse circulant case we saw that the eigenvalue structure is similar to the square root of the periodogram and the maximum of the periodogram is not tight with the scaling $b_{n}^{1 / \alpha}$ when $\alpha \geq 1$ (even with input sequence as i.i.d. S $\alpha$ S random variables). Instead it is tight with a different scaling (see [Mikosch et al.(2000)], Section 3 for details). 


\section{Results for dependent inputs}

Now suppose that the input sequence is a linear process $\left\{X_{t}, t \in \mathbb{Z}\right\}$ given by

$$
X_{t}=\sum_{j=-\infty}^{\infty} a_{j} Z_{t-j}, \quad t \in \mathbb{Z}, \text { where } \sum_{j=-\infty}^{\infty}\left|a_{j}\right|^{\alpha-\epsilon}<\infty \text { for some } 0<\epsilon<\alpha .
$$

Suppose that $\left\{Z_{i}\right\}$ are i.i.d random variables satisfying (1) with $0<\alpha<1$. Using $\mathrm{E}|Z|^{\alpha-\epsilon}<\infty$ and the assumption on the $\left\{a_{j}\right\}$ we have,

$$
\mathrm{E}\left|X_{t}\right|^{\alpha-\epsilon} \leq \sum_{j=-\infty}^{\infty}\left|a_{j}\right|^{\alpha-\epsilon} \mathrm{E}\left|Z_{t-j}\right|^{\alpha-\epsilon}=\mathrm{E}\left|Z_{1}\right|^{\alpha-\epsilon} \sum_{j=-\infty}^{\infty}\left|a_{j}\right|^{\alpha-\epsilon}<\infty
$$

Hence $X_{t}$ is finite a.s. Let

$$
\psi(x)=\sum_{j=-\infty}^{\infty} a_{j} \exp (-i 2 \pi x j), \quad x \in[0,1]
$$

be the transfer function of the linear filter $\left\{a_{j}\right\}$ and $f_{X}(x)$ be the power transfer function of $\left\{X_{t}\right\}$. Then

$$
f_{X}(x)=|\psi(x)|^{2}
$$

Define

$$
\mathrm{M}\left(R C_{n}, f_{X}\right)=\max _{0 \leq k<\frac{n}{2}} \frac{\left|\lambda_{k}\right|}{\sqrt{f_{X}(k / n)}}, \mathrm{M}\left(C_{n}, f_{X}\right)=\max _{0 \leq k<\frac{n}{2}} \frac{\left|\lambda_{k}\right|}{\sqrt{f_{X}(k / n)}}, \mathrm{M}\left(S C_{n}, f_{X}\right)=\max _{0 \leq k<\frac{n}{2}} \frac{\left|\lambda_{k}\right|}{\sqrt{f_{X}(k / n)}}
$$

where in each case $\left\{\lambda_{k}\right\}$ are the eigenvalues of the corresponding matrix. From the eigenvalue structure of $C_{n}$ and $R C_{n}, \mathrm{M}\left(C_{n}, f_{X}\right)=\mathrm{M}\left(R C_{n}, f_{X}\right)$.

Theorem 4. Assume that $\left\{X_{n}\right\}$ and $\left\{a_{j}\right\}$ satisfy (77) and $\left\{Z_{t}\right\}$ is i.i.d satisfying (17). Suppose $f_{X}$ is strictly positive on $[0,1 / 2]$. Then

(a) $M\left(b_{n}^{-1} C_{n}, f_{X}\right) \Rightarrow Y_{\alpha}$ and $M\left(b_{n}^{-1} R C_{n}, f_{X}\right) \Rightarrow Y_{\alpha}$.

(b) Further, if $a_{j}=a_{-j}$ then $M\left(b_{n}^{-1} S C_{n}, f_{X}\right) \Rightarrow 2^{1-1 / \alpha} Y_{\alpha}$.

\section{Proofs of the results}

Some auxiliary results. The main idea of the proofs is taken from [Mikosch et al.(2000)] who show weak convergence of the maximum of the periodogram based on heavy tailed sequence for $\alpha<1$. Let $\epsilon_{x}(\cdot)$ denote the point measure which gives unit mass to any set containing $x$ and let $E=[0,1] \times([-\infty, \infty] \backslash\{0\})$. Let $M_{p}(E)$ be the set of point measures on $E$, topologized by vague convergence. The following convergence result follows from Proposition 3.21 of [Resnick(1987)]:

$$
N_{n}:=\sum_{k=1}^{n} \epsilon_{\left(k / n, Z_{k} / b_{n}\right)} \Rightarrow N:=\sum_{j=1}^{\infty} \epsilon_{\left(U_{j}, B_{j} \Gamma_{j}^{-1 / \alpha}\right)} \text { in } M_{p}(E) .
$$

Suppose $f$ is a bounded continuous complex valued function defined on $\mathbb{R}$ and without loss of generality assume $|f(x)| \leq 1$ for all $x \in \mathbb{R}$. Now pick $\eta>0$ and define $T_{\eta}: M_{p}(E) \longrightarrow C[0, \infty)$ as follows:

$$
\left(T_{\eta} m\right)(x)=\sum_{j} v_{j} 1_{\left\{\left|v_{j}\right|>\eta\right\}} f\left(2 \pi x t_{j}\right)
$$


if $m=\sum_{j} \epsilon_{\left(t_{j}, v_{j}\right)} \in M_{p}(E)$ and $v_{j}$ 's are finite. Elsewhere, set $\left(T_{\eta} m\right)(x)=0$.

In $C[0, \infty)$ the metric is taken to be $\|x(\cdot)-y(\cdot)\|_{\infty}$ where

$$
\|x(\cdot)-y(\cdot)\|_{\infty}=\sum_{n=1}^{\infty} \frac{1}{2^{n}}\left[\|x(\cdot)-y(\cdot)\|_{n} \wedge 1\right], \text { where }\|x(\cdot)-y(\cdot)\|_{n}=\sup _{t \in[0, n]}|x(t)-y(t)| .
$$

The following Lemma was proved in [Mikosch et al.(2000)] (Lemma 2.3) with the function $f(x)=$ $\exp (-i x)$. Same proof works in our case.

Lemma 1. $T_{\eta}: M_{p}(E) \longrightarrow C[0, \infty)$ is continuous a.s. with respect to the distribution of $N$ given in (8).

The proof of the following result is similar to the proof of Proposition 2.2 of [Mikosch et al.(2000)]. We briefly sketch the proof in our case.

Lemma 2. For $0<\alpha<1$, as $n \rightarrow \infty$ the following convergence holds in $C[0, \infty)$ :

$$
J_{n, Z}(x / n):=\sum_{j=1}^{n} \frac{Z_{j}}{b_{n}} f(2 \pi x j / n) \Rightarrow J_{\infty}(x):=\sum_{j=1}^{\infty} B_{j} \Gamma_{j}^{-1 / \alpha} f\left(2 \pi x U_{j}\right), \quad 0 \leq x<\infty .
$$

Proof. Applying Lemma 1 on (8) we have

$$
\begin{aligned}
J_{n, Z}^{(\eta)}(x / n) & :=\sum_{j=1}^{n} \frac{Z_{j}}{b_{n}} f(2 \pi x j / n) 1_{\left\{\left|Z_{j}\right|>\eta b_{n}\right\}} \\
& \Rightarrow \sum_{j=1}^{\infty} B_{j} \Gamma_{j}^{-1 / \alpha} f\left(2 \pi x U_{j}\right) 1_{\left\{\Gamma_{j}^{-1 / \alpha}>\eta\right\}}:=J_{\infty}^{(\eta)}(x) \text { in } C[0, \infty) .
\end{aligned}
$$

Also, as $\eta \rightarrow 0$ by dominated convergence theorem we have

$$
J_{\infty}^{(\eta)}(x) \Rightarrow J_{\infty}(x):=\sum_{j=1}^{\infty} B_{j} \Gamma_{j}^{-1 / \alpha} f\left(2 \pi x U_{j}\right) .
$$

So using Theorem 3.2 of [Billingsley(1999)], the proof will be complete if for any $\epsilon>0$,

$$
\lim _{\eta \rightarrow 0} \limsup _{n \rightarrow \infty} \mathrm{P}\left(\left\|J_{n, Z}^{(\eta)}-J_{n, Z}\right\|_{\infty}>\epsilon\right)=0,
$$

where $\|x(\cdot)-y(\cdot)\|_{\infty}$ is the metric distance in $C[0, \infty)$. Now since $|f(x)| \leq 1$, we have

$$
\begin{aligned}
\lim _{\eta \rightarrow 0} \limsup _{n \rightarrow \infty} \mathrm{P}\left(\left\|J_{n, Z}^{(\eta)}-J_{n, Z}\right\|_{\infty}>\epsilon\right) & \leq \lim _{\eta \rightarrow 0} \limsup _{n \rightarrow \infty} \mathrm{P}\left(\sum_{j=1}^{n}\left|\frac{Z_{j}}{b_{n}}\right| 1_{\left\{\left|Z_{j}\right| \leq \eta b_{n}\right\}}>\epsilon\right) \\
& \leq \lim _{\eta \rightarrow 0} \limsup _{n \rightarrow \infty} n \epsilon^{-1} \mathrm{E}\left(\left|\frac{Z_{1}}{b_{n}}\right| 1_{\left\{\left|Z_{j}\right| \leq \eta b_{n}\right\}}\right) .
\end{aligned}
$$

By an application of Karamata's theorem (see [Resnick(1987)] Exercise 0.4.2.8) we get

$$
n \mathrm{E}\left(\left|\frac{Z_{1}}{b_{n}}\right| 1_{\left\{\left|Z_{j}\right| \leq \eta b_{n}\right\}}\right) \quad \sim_{n \rightarrow \infty} \frac{\alpha}{1-\alpha} n \eta \mathrm{P}\left(\left|Z_{1}\right|>\eta b_{n}\right) \sim_{n \rightarrow \infty} \frac{\alpha}{1-\alpha} \eta^{1-\alpha}
$$

and $\frac{\alpha}{1-\alpha} \eta^{1-\alpha} \rightarrow 0$ as $\eta \rightarrow 0$. This completes the proof of the lemma. 
Proof of Theorem 1. We use Lemma 1 and 2 with $f(x)=\exp (-i x)$. It is immediate that

$$
b_{n}^{-1}\left\|C_{n}\right\| \leq b_{n}^{-1} \sum_{t=1}^{n}\left|Z_{t}\right|
$$

It is well known that

$$
b_{n}^{-1} \sum_{t=1}^{n}\left|Z_{t}\right| \Rightarrow Y_{\alpha}=\sum_{j=1}^{\infty} \Gamma_{j}^{-1 / \alpha} \sim S_{\alpha}\left(C_{\alpha}^{-1 / \alpha}, 1,0\right) .
$$

Hence it remains to show that for $\gamma>0$,

$$
\liminf _{n \rightarrow \infty} \mathrm{P}\left(b_{n}^{-1}\left\|C_{n}\right\|>\gamma\right) \geq \mathrm{P}\left(Y_{\alpha}>\gamma\right)
$$

Now observe that for any integer $K$ and sufficiently large $n$,

$$
\mathrm{P}\left(\sup _{j=1, \ldots,[n / 2]}\left|J_{n, Z}(j / n)\right|>\gamma\right) \geq \mathrm{P}\left(\sup _{j=1, \ldots, K}\left|J_{n, Z}(j / n)\right|>\gamma\right) .
$$

Now from Lemma 2 we have

$$
\left(J_{n, Z}(j / n), 1 \leq j \leq K\right) \Rightarrow\left(J_{\infty}(j), 1 \leq j \leq K\right)
$$

in $\mathbb{R}^{K}$. Hence

$$
\sup _{j=1, \ldots, K}\left|J_{n, Z}(j / n)\right| \Rightarrow \sup _{j=1, \ldots, K}\left|J_{\infty}(j)\right|
$$

and so letting $K \rightarrow \infty$,

$$
\liminf _{n \rightarrow \infty} \mathrm{P}\left(\sup _{j=1, \ldots,[n / 2]}\left|J_{n, Z}(j / n)\right|>\gamma\right) \geq \mathrm{P}\left(\sup _{j=1, \ldots, \infty}\left|J_{\infty}(j)\right|>\gamma\right)
$$

Now the theorem follows from Lemma 3 given below.

This lemma is similar to Lemma 2.4 of [Mikosch et al.(2000)] and hence we skip the proof.

\section{Lemma 3.}

$$
\sup _{j=1, \ldots, \infty}\left|J_{\infty}(j)\right|=\sup _{j=1, \ldots, \infty}\left|\sum_{t=1}^{\infty} B_{t} \Gamma_{t}^{-1 / \alpha} \exp \left(-2 \pi i j U_{t}\right)\right|=Y_{\alpha} \text { a.s. }
$$

Proof of Theorem 2, The proof is similar to the proof of Theorem 1. We provide the proof for $n$ odd, and for $n$ even the changes needed are minor. Define

$$
J_{n, Z}(x):=2 b_{n}^{-1} \sum_{t=1}^{q} Z_{t} \cos (2 \pi x t) \text { and } M_{n, Z}:=\max _{0 \leq k \leq q}\left|J_{n, Z}(k / n)\right|
$$

where $q=q_{n}=\left[\frac{n}{2}\right]$. Since ||$\left|b_{n}^{-1} S C_{n} \|-M_{n, Z}\right| \rightarrow 0$ almost surely, it is enough to show $M_{n, Z} \Rightarrow$ $2^{1-1 / \alpha} Y_{\alpha}$. Note that 8 holds with $[0,1]$ replaced by $[0,1 / 2]$, and letting $N_{n}=\sum_{k=1}^{q} \epsilon_{\left(k / n, Z_{k} / b_{q}\right)}$, $N=\sum_{j=1}^{\infty} \epsilon_{\left(U_{j}, B_{j} \Gamma_{j}^{-1 / \alpha}\right)}$ and $U_{j}$ to be i.i.d. $U[0,1 / 2]$. Now following the argument given in Lemma 2.3 of [Mikosch et al.(2000)], Lemma 2 and taking $f(x)=\cos x$ it is easy to establish that

$$
J_{n, Z}(x / n)=2 b_{n}^{-1} \sum_{k=1}^{q} Z_{k} \cos \frac{2 \pi k x}{n} \Rightarrow 2^{1-1 / \alpha} \sum_{j=1}^{\infty} B_{j} \Gamma_{j}^{-1 / \alpha} \cos \left(2 \pi x U_{j}\right):=J_{\infty}(x) .
$$


It is obvious that

$$
M_{n, Z} \leq 2 b_{n}^{-1} \sum_{t=1}^{q}\left|Z_{t}\right| \Rightarrow 2^{1-1 / \alpha} \sum_{j=1}^{\infty} \Gamma_{j}^{-1 / \alpha}=2^{1-1 / \alpha} Y_{\alpha} .
$$

It remains to show that for $\eta>0$,

$$
\liminf _{n \rightarrow \infty} \mathrm{P}\left(M_{n, Z}>\eta\right) \geq \mathrm{P}\left(2^{1-1 / \alpha} Y_{\alpha}>\eta\right) .
$$

Now following the arguments given to prove (12), we can establish this relation. This completes the proof of the theorem.

Proof of Theorem 3, Following [Meckes(2007)], $T_{n}$ is a submatrix of the infinite Laurent matrix

$$
L_{n}=\left[Z_{|j-k|} 1_{|j-k| \leq n-1}\right]_{j, k \in \mathbb{Z}}
$$

so $\left\|T_{n}\right\| \leq\left\|L_{n}\right\|$, where $\left\|L_{n}\right\|$ denotes the operator norm of $L_{n}$ acting in the standard way on $l_{2}(\mathbb{Z})$. If we use the Fourier basis to identify $l_{2}(\mathbb{Z})$ with $L_{2}[0,1]$, it turns out that $L_{n}$ corresponds to a multiplication operator with the multiplier

$$
g(x)=\sum_{j=-(n-1)}^{n-1} Z_{|j|} e^{2 \pi i j x}=Z_{0}+2 \sum_{j=1}^{n-1} \cos (2 \pi j x) Z_{j} .
$$

Therefore

$$
\left\|T_{n}\right\| \leq\left\|L_{n}\right\|=\|g\|_{\infty}=\sup _{0 \leq x \leq 1}|g(x)|
$$

Hence as $n \rightarrow \infty$,

$$
b_{n}^{-1}\left\|T_{n}\right\| \leq b_{n}^{-1}\left[\left|Z_{0}\right|+2 \sum_{j=0}^{n-1}\left|Z_{j}\right|\right] \Rightarrow 2 \sum_{j=1}^{\infty} \Gamma_{j}^{-1 / \alpha}
$$

and we have for $\gamma>0$

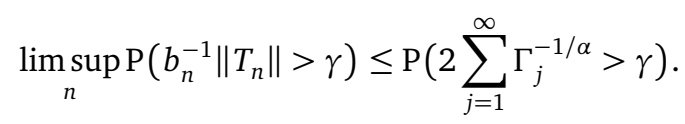

By another argument of [Meckes(2007)], we get the following estimate

$$
\left\|T_{n}\right\|=\sup _{v \in \mathbb{C}^{n} \backslash\{0\}} \frac{\left\langle T_{n} v, v\right\rangle}{\langle v, v\rangle} \geq \sup _{0 \leq x \leq 1} \frac{1}{n}\left|\left\langle T_{n} v_{x}, v_{x}\right\rangle\right|,
$$

where $v_{x} \in \mathbb{C}^{n}$ is defined as $\left(v_{x}\right)_{j}=e^{2 \pi i x j}$ for $j=1,2, \ldots, n$ and $\langle\cdot, \cdot\rangle$ is the standard inner product on $\mathbb{C}^{n}$. Therefore

$$
\begin{aligned}
\left\|T_{n}\right\| & \geq \frac{1}{n} \sup _{0 \leq x \leq 1}\left|\sum_{j, k=1}^{n} Z_{|j-k|} e^{2 \pi i(j-k) x}\right| \\
& =\frac{1}{n} \sup _{0 \leq x \leq 1}\left|\sum_{j=-(n-1)}^{n-1}(n-|j|) Z_{|j|}\right|^{2 \pi i j x} \mid \\
& =\sup _{0 \leq x \leq 1}\left|Z_{0}+2 \sum_{j=1}^{n-1}\left(1-\frac{j}{n}\right) Z_{j} \cos (2 \pi j x)\right| .
\end{aligned}
$$


Now

$$
\begin{aligned}
\operatorname{liminfP}_{n}\left(b_{n}^{-1}\left\|T_{n}\right\|>\gamma\right) & \geq \operatorname{liminfP}_{n}\left(b_{n}^{-1} \sup _{0 \leq x \leq 1}\left|Z_{0}+2 \sum_{j=1}^{n-1}\left(1-\frac{j}{n}\right) Z_{j} \cos (2 \pi j x)\right|>\gamma\right) \\
& =\lim _{n} \mathrm{P}\left(b_{n}^{-1} \sup _{0 \leq x \leq 1}\left|2 \sum_{j=1}^{n-1}\left(1-\frac{j}{n}\right) Z_{j} \cos (2 \pi j x)\right|>\gamma\right)
\end{aligned}
$$

To find the limit in the last expression, pick $\eta>0$ and define $T_{\eta}: M_{p}(E) \longrightarrow C[0, \infty)$, as follows:

$$
\left(T_{\eta} m\right)(x)= \begin{cases}\sum_{j}\left(1-t_{j}\right) v_{j} \cos \left(2 \pi x t_{j}\right) 1\left(\left|v_{j}\right|>\eta\right) & \text { if } m=\sum_{j} \epsilon_{\left(t_{j}, v_{j}\right)}, \text { all } v_{j}^{\prime} s \text { are finite } \\ 0 & \text { otherwise }\end{cases}
$$

Following the argument given in Lemma 2.3 of [Mikosch et al.(2000)], it is easy to see that $T_{\eta}$ is continuous a.s. with respect to the distribution of $N$ and then using an argument from Lemma 2 , we can show that for fixed $x$

$$
2 b_{n}^{-1} \sum_{j=1}^{n-1}(1-j / n) Z_{j} \cos (2 \pi j x / n) \Rightarrow 2 \sum_{j=1}^{\infty}\left(1-U_{j}\right) B_{j} \Gamma_{j}^{-1 / \alpha} \cos \left(2 \pi x U_{j}\right) .
$$

Now for any fixed $T$ where $n>T>0$, using $[15)$,

$$
\begin{aligned}
\sup _{0 \leq x \leq 1}\left|2 b_{n}^{-1} \sum_{j=1}^{n-1}\left(1-\frac{j}{n}\right) Z_{j} \cos (2 \pi j x)\right| & =\sup _{0 \leq x \leq n}\left|2 b_{n}^{-1} \sum_{j=1}^{n-1}\left(1-\frac{j}{n}\right) Z_{j} \cos \frac{2 \pi j x}{n}\right| \\
& \geq \sup _{0 \leq x \leq T}\left|2 b_{n}^{-1} \sum_{j=1}^{n-1}\left(1-\frac{j}{n}\right) Z_{j} \cos \frac{2 \pi j x}{n}\right| \\
& \Rightarrow \sup _{0 \leq x \leq T}\left|2 \sum_{j=1}^{\infty}\left(1-U_{j}\right) B_{j} \Gamma_{j}^{-1 / \alpha} \cos \left(2 \pi x U_{j}\right)\right|,
\end{aligned}
$$

and hence

$$
\liminf _{n} \mathrm{P}\left(b_{n}^{-1}\left\|T_{n}\right\|>\gamma\right) \geq \mathrm{P}\left(\sup _{0 \leq x \leq T}\left|2 \sum_{j=1}^{\infty}\left(1-U_{j}\right) B_{j} \Gamma_{j}^{-1 / \alpha} \cos \left(2 \pi x U_{j}\right)\right|>\gamma\right) .
$$

Since this is true for any $T$, we obtain

$$
\liminf _{n} \mathrm{P}\left(b_{n}^{-1}\left\|T_{n}\right\|>\gamma\right) \geq \mathrm{P}\left(\sup _{0 \leq x<\infty}\left|2 \sum_{j=1}^{\infty}\left(1-U_{j}\right) B_{j} \Gamma_{j}^{-1 / \alpha} \cos \left(2 \pi x U_{j}\right)\right|>\gamma\right) .
$$

Now to identify the distribution of the random variable appearing in the right side of the inequality, we follow Lemma 2.4 of [Mikosch et al.(2000)]. Here we use the fact

$$
\left\{\left(\overline{x U_{1}(\omega)}, \ldots, \overline{x U_{m}(\omega)}\right), x \geq 0\right\}
$$

is dense in $[0,1]^{m}$ and we get

$$
\sup _{0 \leq x<\infty}\left|2 \sum_{j=1}^{\infty}\left(1-U_{j}\right) B_{j} \Gamma_{j}^{-1 / \alpha} \cos \left(2 \pi x U_{j}\right)\right| \Rightarrow \sum_{j=1}^{\infty}\left(1-U_{j}\right) \Gamma_{j}^{-1 / \alpha} .
$$

This completes the proof. 
Remark 3. The assumption of $\alpha<1$ is crucially used only in the lower bound argument. It is clear from the above proof that the upper bound can be derived when $\alpha \in(1,2)$. Indeed, it easily follows that

$$
\limsup _{n \rightarrow \infty} P\left(\frac{\left\|T_{n}\right\|-2 n \mathrm{E}\left[\left|Z_{1}\right|\right]}{b_{n}}>x\right) \leq P\left(2 \widetilde{Y_{\alpha}}>x\right)
$$

where $\widetilde{Y_{\alpha}}$ is as in Remark 1(ii).

Proof of Theorem 4(a). The proof is along the lines of the proof of Lemma 2.6 in [Mikosch et al.(2000)]. Let $\widehat{C_{n}}$ be the circulant matrix formed with independent entries $\left\{Z_{i}\right\}$. To prove the result it is enough to show that

$$
\left|\mathrm{M}\left(b_{n}^{-1} C_{n}, f_{X}\right)-\left\|b_{n}^{-1} \widehat{C_{n}}\right\|\right| \stackrel{\mathscr{P}}{\longrightarrow} 0 .
$$

Let $J_{n, Z}(x)=b_{n}^{-1} \sum_{t=1}^{n} Z_{t} \exp (-i 2 \pi x t)$. Note

$$
\begin{aligned}
\left|\operatorname{M}\left(b_{n}^{-1} C_{n}, f_{X}\right)-\left\|b_{n}^{-1} \widehat{C_{n}}\right\|\right| & =\left|\sup _{1 \leq k \leq n}\left(f_{X}(k / n)\right)^{-1 / 2}\right| J_{n, X}(k / n)\left|-\sup _{1 \leq k \leq n}\right| J_{n, Z}(k / n)|| \\
& \leq \sup _{1 \leq k \leq n}|| \psi(k / n)^{-1} J_{n, X}(k / n)|-| J_{n, Z}(k / n)|| \\
& \leq \sup _{1 \leq k \leq n}\left|\psi(k / n)^{-1} J_{n, X}(k / n)-J_{n, Z}(k / n)\right|
\end{aligned}
$$

and

$$
\begin{aligned}
J_{n, X}(x) & =b_{n}^{-1} \sum_{t=1}^{n} X_{t} \exp (-i 2 \pi x t) \\
& =b_{n}^{-1} \sum_{j=-\infty}^{\infty} a_{j} \exp (-i 2 \pi x j)\left(\sum_{t=1}^{n} Z_{t} \exp (-i 2 \pi x t)+V_{n, j}\right) \\
& =\psi(x) J_{n, Z}(x)+Y_{n}(x)
\end{aligned}
$$

where

$$
V_{n, j}=\sum_{t=1-j}^{n-j} Z_{t} \exp (-i 2 \pi x t)-\sum_{t=1}^{n} Z_{t} \exp (-i 2 \pi x t), Y_{n}(x)=b_{n}^{-1} \sum_{j=-\infty}^{\infty} a_{j} \exp (-i 2 \pi x j) V_{n, j} .
$$

Since $f_{X}$ is bounded away from 0 and $(16)$ holds, it is enough to show that $\max _{1 \leq k \leq n}\left|Y_{n}(k / n)\right| \stackrel{\mathscr{P}}{\rightarrow} 0$. Now

$$
\begin{aligned}
Y_{n}(x)= & b_{n}^{-1} \sum_{j=n+1}^{\infty} a_{j} \exp (-i 2 \pi x j) V_{n, j}+b_{n}^{-1} \sum_{j=1}^{n} a_{j} \exp (-i 2 \pi x j) V_{n, j} \\
& +b_{n}^{-1} \sum_{j=-\infty}^{-n-1} a_{j} \exp (-i 2 \pi x j) V_{n, j}+b_{n}^{-1} \sum_{j=-n}^{-1} a_{j} \exp (-i 2 \pi x j) V_{n, j} \\
= & S_{1}(x)+S_{2}(x)+S_{3}(x)+S_{4}(x) .
\end{aligned}
$$

Now following an argument similar to that given in the proof of Lemma 2.6 in [Mikosch et al.(2000)], we can show that

$$
\max _{1 \leq k \leq n}\left|S_{i}(k / n)\right| \stackrel{\mathscr{P}}{\rightarrow} 0 \text { for } i=1,2 .
$$


The behavior of $S_{3}(x)$ and $S_{4}(x)$ are similar to $S_{1}(x)$ and $S_{2}(x)$ respectively. Therefore, following similar argument we can show that $\max _{1 \leq k \leq n}\left|S_{j}(k / n)\right| \stackrel{\mathscr{P}}{\rightarrow} 0$ for $j=3,4$. This completes the proof of part (a).

Proof of Theorem 4 (b). Let $\widehat{S C_{n}}$ be the symmetric circulant matrix formed with independent entries $\left\{Z_{i}\right\}$. In view of Theorem 2, it is enough to show that

$$
\left|\mathrm{M}\left(b_{n}^{-1} S C_{n}, f_{X}\right)-\left\|b_{n}^{-1} \widehat{S C_{n}}\right\|\right| \stackrel{\mathscr{P}}{\rightarrow} 0
$$

Let $q=q_{n}=\left[\frac{n}{2}\right]$ and

$$
\begin{aligned}
J_{n, Z}(x) & :=2 b_{n}^{-1} \sum_{t=1}^{q} Z_{t} \cos (2 \pi x t) \\
& =b_{n}^{-1} \sum_{t=1}^{q} Z_{t} \exp (i 2 \pi x t)+b_{n}^{-1} \sum_{t=1}^{q} Z_{t} \exp (-i 2 \pi x t)
\end{aligned}
$$

Then using $a_{j}=a_{-j}$ we have

$$
\begin{aligned}
J_{n, X}(x):= & b_{n}^{-1} \sum_{t=1}^{q} X_{t} \exp (i 2 \pi x t)+b_{n}^{-1} \sum_{t=1}^{q} X_{t} \exp (-i 2 \pi x t) \\
= & b_{n}^{-1} \sum_{j=-\infty}^{\infty} a_{j} \exp (-i 2 \pi x j)\left(\sum_{t=1}^{q} Z_{t} \exp (i 2 \pi x t)+U_{n, j}\right) \\
& +b_{n}^{-1} \sum_{j=-\infty}^{\infty} a_{j} \exp (-i 2 \pi x j)\left(\sum_{t=1}^{q} Z_{t} \exp (-i 2 \pi x t)+V_{n, j}\right) \\
= & \psi(x) J_{n, Z}(x)+Y_{1 n}(x)+Y_{2 n}(x),
\end{aligned}
$$

where

$$
\begin{gathered}
U_{n, j}=\sum_{t=1+j}^{q+j} Z_{t} \exp (i 2 \pi x t)-\sum_{t=1}^{q} Z_{t} \exp (i 2 \pi x t), V_{n, j}=\sum_{t=1-j}^{q-j} Z_{t} \exp (-i 2 \pi x t)-\sum_{t=1}^{q} Z_{t} \exp (-i 2 \pi x t), \\
Y_{1 n}=b_{n}^{-1} \sum_{j=-\infty}^{\infty} a_{j} \exp (-i 2 \pi x j) U_{n, j}, \quad Y_{2 n}=b_{n}^{-1} \sum_{j=-\infty}^{\infty} a_{j} \exp (-i 2 \pi x j) V_{n, j} .
\end{gathered}
$$

Since $f_{X}$ is bounded away from 0 , it is enough to show that

$$
\sup _{1 \leq k \leq q}\left|J_{n, X}(k / n)-\psi(k / n) J_{n, Z}(k / n)\right| \leq \sup _{1 \leq k \leq q}\left|Y_{1 n}(k / n)\right|+\sup _{1 \leq k \leq q}\left|Y_{2 n}(k / n)\right| \stackrel{\mathscr{P}}{\rightarrow} 0 .
$$


Now

$$
\begin{aligned}
Y_{1 n}(x)= & b_{n}^{-1} \sum_{j=-\infty}^{\infty} a_{j} \exp (-i 2 \pi x j) U_{n, j} \\
= & b_{n}^{-1} \sum_{j=q+1}^{\infty} a_{j} \exp (-i 2 \pi x j) U_{n, j}+b_{n}^{-1} \sum_{j=1}^{q} a_{j} \exp (-i 2 \pi x j) U_{n, j} \\
& +b_{n}^{-1} \sum_{j=-\infty}^{-q-1} a_{j} \exp (-i 2 \pi x j) U_{n, j}+b_{n}^{-1} \sum_{j=-q}^{-1} a_{j} \exp (-i 2 \pi x j) U_{n, j} \\
= & S_{1}(x)+S_{2}(x)+S_{3}(x)+S_{4}(x) .
\end{aligned}
$$

Again following an argument similar to that in the proof of Lemma 2.6 in [Mikosch et al.(2000)], we can show that $\sup _{1 \leq k \leq q}\left|S_{i}(k / n)\right| \stackrel{\mathscr{P}}{\rightarrow} 0$ for $1 \leq i \leq 4$. Hence $\sup _{1 \leq k \leq q}\left|Y_{1 n}(k / n)\right| \stackrel{\mathscr{P}}{\rightarrow} 0$. Similarly $\sup _{1 \leq k \leq q}\left|Y_{2 n}(k / n)\right| \stackrel{\mathscr{P}}{\rightarrow} 0$. This completes the proof of part (b).

Acknowledgement. We thank the two anonymous Referees whose detailed and constrictive comments have led to a significant improvement in the presentation.

\section{References}

[Adamczak(2010)] Adamczak, Radosław. A few remarks on the operator norm of random Toeplitz matrices. J. Theoret. Probab., 23 no. 1, 85-108, 2010. MR2591905

[Auffinger et al.(2009)] Auffinger, Antonio, Ben Arous, Gerard and Peche, Sandrine. Poisson convergence for the largest eigenvalues of Heavy Tailed Random Matrices. Annales de l'Institut Henri Poincare., 45, no. 3, 859-610, 2009. MR2548495

[Bai(1999)] Bai, Z. D. Methodologies in spectral analysis of large dimensional random matrices, a review. Statistica Sinica, 9, 611-677, 1999 (with discussions). MR1711663

[Bai and Yin (1988)] Bai, Z.D and Yin, Y.Q. Necessary and sufficient conditions for almost sure convergence of the largest eigenvalue of a Wigner matrix. Ann. Probab., 16, no. 4, 1729-1741, 1988. MR0958213

[Basak and Bose(2009)] Basak, Anirban and Bose, Arup. Limiting spectral distribution of some band matrices. Technical Report R16/2009, Stat-Math Unit, Indian Statistical Institute, Kolkata. To appear in Periodica Hungarica, 2010.

[Billingsley(1999)] Billingsley, Patrick. Convergence of probability measures. Wiley Series in Probability and Statistics: Probability and Statistics. Second edition, 1999. MR1700749

[Bose et al.(2009a)] Bose, Arup; Hazra, Rajat Subhra and Saha, Koushik. Limiting spectral distribution of circulant type matrices with dependent inputs. Electron. J. Probab., 14, no. 86, 2463-2491, 2009a. MR2563248

[Bose et al.(2009b)] Bose, Arup; Hazra, Rajat Subhra and Saha, Koushik. Spectral norm of circulant type matrices. To appear in J. Theoret. Probab. DOI 10.1007/s10959-009-0257-5. 2009b. 
[Bose and Mitra(2002)] Bose, Arup and Mitra, Joydip. Limiting spectral distribution of a special circulant. Statist. Probab. Lett., 60(1), 111-120, 2002. MR1945684

[Bose and Sen(2007)] Bose, Arup and Sen, Arnab. Spectral norm of random large dimensional noncentral Toeplitz and Hankel matrices. Electron. Comm. Probab., 12, 29-35, 2007. MR2284045

[Bryc, Dembo and Jiang (2006)] Włodzimierz Bryc, Amir Dembo and, Tiefeng Jiang. Spectral measure of large random Hankel, Markov and Toeplitz matrices. Ann. Probab., 34(1), 1-38, 2006. MR2206341

[Bryc and Sethuraman(2009)] Bryc, Wlodek and Sethuraman, Sunder. A remark on maximum eigenvalue for circulant matrices. High Dimensional Probabilities V: The Luminy Volume, IMS Collections Vol. 5, 179-184, 2009.

[Davis and Mikosch(1999)] Davis, Richard A. and Mikosch, Thomas. The maximum of the periodogram of a non-Gaussian sequence. Ann. Probab., 27, no. 1, 522-536, 1999. MR1681157

[Fan and Yao(2003)] Jianqing Fan and Qiwei Yao. Nonlinear time series. Springer Series in Statistics. Springer-Verlag, New York, 2003.

[Gray (2006)] Gray, Robert M. Toeplitz and Circulant Matrices: A review. Now Publishers, Norwell, Massachusetts, 2006.

[Grenander and Szegő(2001)] Grenander, U. and Szegő, G. Toeplitz forms and their applications Chelsea Pub. Co. 2001. MR0890515

[Hammond and Miller(2005)] Hammond, Christopher and Miller, Steven J. Distribution of eigenvalues for the ensemble of real symmetric Toeplitz matrices. J. Theoret. Probab., 18, no. 3, 537-566, 2005. MR2167641

[Kargin(2009)] Kargin, Vladislav. Spectrum of random Toeplitz matrices with band structures. Elect. Comm. in Probab. 14, 412-421, 2009. MR2551851

[Lin and Liu(2009)] Lin, Zhengyan and Liu, Weidong. On maxima of periodograms of stationary processes. Ann. Statist., 37, no. 5B, 2676-2695, 2009. MR2541443

[Massey et al.(2007)] Massey, A., Miller, S.J. and Sinsheimer, J. Distribution of eigenvalues of real symmetric palindromic Toeplitz matrices and circulant matrices. J. Theoret. Probab. 20, no. 3, 637-662, 2007. MR2337145

[Meckes(2007)] Meckes, Mark W. On the spectral norm of a random Toeplitz matrix. Electron. Comm. Probab. 12, 315-325, 2007. MR2342710

[Meckes (2009)] Meckes, Mark W. Some results on random circulant matrices. High Dimensional Probability V: The Luminy Volume, 213-223, IMS Collections 5, Institute of Mathematical Statistics, Beachwood, OH, 2009.

[Mikosch et al.(2000)] Mikosch, Thomas and Resnick, Sidney and Samorodnitsky, Gennady. The maximum of the periodogram for a heavy-tailed sequence. Ann. Probab., 28, no. 2, 885-908, 2000. MR1782277 
[Resnick(1987)] Resnick, Sidney I. Extreme values, regular variation, and point processes. Applied Probability. A Series of the Applied Probability Trust. Springer-Verlag, New York, 1987. MR0900810

[Samorodnitsky and Taqqu (1994)] Samorodnitsky, Gennady and Taqqu, Murad S. Stable nonGaussian random processes. Chapman \& Hall, New York, 1994. MR1280932

[Silverstein(1994)] Silverstein, Jack W. The spectral radii and norms of large-dimensional noncentral random matrices. Comm. Statist. Stochastic Models, 10, no. 3, 525-532, 1994. MR1284550

[Soshnikov (2004)] Soshnikov, Alexander. Poisson statistics for the largest eigenvalues of Wigner random matrices with heavy tails. Electron. Comm. Probab, 9, 82-91, 2004. MR2081462

[Soshnikov (2006)] Soshnikov, Alexander. Poisson statistics for the largest eigenvalues in random matrix ensembles. Mathematical physics of quantum mechanics, 351-364. Lecture Notes in Phys., 690, Springer, Berlin, 2006. MR2234922

[Yin et al.(1988)] Yin, Y.Q., Bai, Z.D. and Krishnaiah, P.R. On the limit of the largest eigenvalue of the large dimensional sample covariance matrix. Probab. Theor. Relat. Field, 78, no. 4, 509-521, 1988. MR0950344 